\title{
Long-Term Neuropathological Changes Associated with Cerebral Palsy in a Nonhuman Primate Model of Hypoxic-Ischemic Encephalopathy
}

\author{
Ryan M. McAdams ${ }^{a}$ Bobbi Fleiss ${ }^{\mathrm{e}}$ f Christopher Traudt $^{\mathrm{a}}$ \\ Leslie Schwendimann $^{e}{ }^{f}$ Jessica M. Snyder ${ }^{c}$ Robin L. Haynes ${ }^{d}$ \\ Niranjana Natarajan ${ }^{b}$ Pierre Gressens ${ }^{\text {e, }}{ }^{\text {Sandra E. Juul }}{ }^{\mathrm{a}}$ \\ a Division of Neonatology, Department of Pediatrics, ${ }^{b}$ Division of Pediatric Neurology, Department of Neurology, \\ and 'Department of Comparative Medicine, University of Washington, Seattle, WA, and d Department of Pathology, \\ Boston Children's Hospital, Boston, MA, USA; 'PROTECT, INSERM, Université Paris Diderot, Sorbonne Paris Cité, \\ Paris, France; ${ }^{f}$ Department of Perinatal Imaging and Health, Imaging Sciences and Biomedical Engineering Research \\ Division at King's College London, King's Health Partners, St Thomas' Hospital, London, UK
}

\section{Keywords}

Brain injury · Central nervous system · Cerebellum ·

Developing brain - Erythropoietin · Hypothermia therapy ·

Hypoxic-ischemic encephalopathy - Immunohistochemistry · Monkey

\begin{abstract}
Background: Cerebral palsy (CP) is the most common motor disability in childhood, with a worldwide prevalence of 1.54/1,000 live births. Hypoxic-ischemic encephalopathy (HIE) contributes to the burden of $\mathrm{CP}$, but the long-term neuropathological findings of this association remain limited. Methodology: Thirty-four term Macaca nemestrina macaques were included in this long-term neuropathological study: 9 control animals delivered by cesarean section and 25 animals with perinatal asphyxia delivered by cesarean section after 15-18 min of umbilical cord occlusion (UCO). UCO animals were randomized to saline $(n=11)$, therapeutic
\end{abstract}

\section{KARGER}

(C) 2017 S. Karger AG, Basel

E-Mail karger@karger.com

www.karger.com/dne hypothermia (TH; $n=6)$, or $\mathrm{TH}+$ erythropoietin (Epo; $n=8)$. Epo was given on days 1, 2, 3, and 7. Animals had serial developmental assessments and underwent magnetic resonance imaging with diffusion tensor imaging at 9 months of age followed by necropsy. Histology and immunohistochemical (IHC) staining of brain and brainstem sections were performed. Results: All UCO animals demonstrated and met the standard diagnostic criteria for human neonates with moderate-to-severe HIE. Four animals developed moderateto-severe CP (3 UCO and $1 \mathrm{UCO}+\mathrm{TH}$ ), 9 had mild CP (2 UCO, $3 \mathrm{UCO}+\mathrm{TH}, 3 \mathrm{UCO}+\mathrm{TH}+$ Epo, and 1 control), and $2 \mathrm{UCO}$ animals died. None of the animals treated with $\mathrm{TH}+$ Epo died, had moderate-to-severe $\mathrm{CP}$, or demonstrated signs of long-term neuropathological toxicity. Compared to animals grouped together as having no CP (no-CP; controls and mild CP only), animals with CP (moderate and severe) demon-

Ryan M. McAdams and Bobbi Fleiss are joint first authors.
Sandra E. Juul, MD, $\mathrm{PhD}$

Division of Neonatology, Department of Pediatrics, University of Washington 1959 NE Pacific Street, HSB RR542D, UW Box 356320 Seattle, WA 98195-6320 (USA)

E-Mailsjuul@uw.edu 
strated decreased fractional anisotropy of multiple whitematter tracts including the corpus callosum and internal capsule, when using Tract-Based Spatial Statistics (TBSS). Animals with $\mathrm{CP}$ had decreased staining for cortical neurons and increased brainstem glial scarring compared to animals without CP. The cerebellar cell density of the internal granular layer and white matter was decreased in CP animals compared to that in control animals without CP. Conclusions/ Significance: In this nonhuman primate HIE model, animals treated with $\mathrm{TH}+$ Epo had less brain pathology noted on TBSS and IHC staining, which supports the long-term safety of TH + Epo in the setting of HIE. Animals that developed CP showed white-matter changes noted on TBSS, subtle histopathological changes in both the white and gray matter, and brainstem injury that correlated with CP severity. This HIE model may lend itself to further study of the relationship between brainstem injury and CP.

(c) 2017 S. Karger AG, Basel

\section{Introduction}

Intrapartum hypoxia is one important cause of hypoxic-ischemic encephalopathy (HIE), a problem that contributes to $22 \%$ of neonatal deaths worldwide $[1,2]$. Common causes of intrapartum hypoxia are intrauterine asphyxia brought on by circulatory problems, such as the clotting of placental arteries, placental abruption, or inflammatory processes [3]. In developed countries, therapeutic hypothermia (TH) initiated within $6 \mathrm{~h}$ of birth is the standard of care for term and near-term neonates with moderate or severe HIE [4-7], and it results in fewer deaths and better neurodevelopmental outcomes up to at least 18 months of age for survivors when compared to untreated neonates [8]. Despite TH, $46 \%$ of treated neonates with HIE still die or sustain major neurodevelopmental disability, with approximately $20 \%$ developing cerebral palsy (CP) [4-12]. Since TH only provides a partial treatment benefit, adjunctive therapies are needed. Erythropoietin (Epo), a hematopoietic cytokine with neuroprotective effects, is a promising treatment for neonates with HIE $[13,14]$ that could be used concurrently with TH $[15,16]$.

To better understand treatment strategies that may improve outcomes in neonates with HIE (e.g., TH + Epo), we developed a nonhuman primate model of acute perinatal asphyxia using umbilical cord occlusion (UCO) prior to delivery to produce moderate-to-severe HIE $[17,18]$. In our model, UCO for 15-18 min results in clinical and laboratory manifestations of HIE compa-

CP-Associated Neuropathological

Changes in a Primate Model of HIE rable to human newborns entered in the major TH clinical trials $[4,5]$. In addition to early physical exam and magnetic resonance (MR) spectroscopy findings consistent with moderate-to-severe HIE, animals demonstrate transient kidney and liver biochemical dysfunction, abnormal amplitude-integrated electroencephalogram (aEEG), feeding intolerance, progressive spasticity, and cognitive delay $[17,18]$. We found that UCO for $15-18$ min resulted in death or moderate-to-severe CP in $43 \%$ of saline-treated and $44 \%$ of TH-treated animals, but not in any of the animals treated with $\mathrm{TH}+$ Epo [18]. Compared to treatment with $\mathrm{UCO}+$ saline, animals treated with $\mathrm{UCO}+\mathrm{TH}+$ Epo had improved motor and cognitive responses, cerebellar growth, and diffusion tensor imaging (DTI) measurements on MR imaging (MRI). These findings suggest that Epo treatment may play a key role in preventing neurodevelopmental impairment following HIE.

Long-term neuropathological findings following perinatal asphyxia are limited. We have previously reported on 4 animals exposed to UCO for $15 \mathrm{~min}$ that had evidence of neuronal degeneration with gliosis, but extensive immunohistochemistry of normal versus injured or treated animals was not available [17]. Here, we present long-term neuropathological findings from a proof-ofconcept, open-label, randomized controlled study to evaluate the incidence of death or CP by treatment group. We also provide a secondary analysis of the neuropathology of animals with and without CP to improve our understanding of the underlying neuropathological substrates of this injury.

\section{Methods and Animals}

Animals

The parent study testing the safety and efficacy of $\mathrm{TH}+$ Epo for improving survival and preventing the development of moderate-to-severe CP after HIE in a term nonhuman primate model of perinatal asphyxia included 56 Macaca nemestrina (pigtailed macaques) randomized to different treatment groups [18]. This study focused on brain histopathology at 9 months of age, which was available in 34 animals (25 with HIE and 9 non-UCO control animals delivered by cesarean section). Figure 1 provides an overview of the experimental UCO model used to produce HIE in M. nemestrina. The Animal Care and Use Committees at the University of Washington, in accordance with US National Institutes of Health (NIH), guidelines approved all experimental protocols.

Delivery and Resuscitation

Animals were delivered $1-8$ days prior to term (168 \pm 2 days) by hysterotomy under maternal general anesthesia with sevoflu-

Dev Neurosci 2017;39:124-140

DOI: $10.1159 / 000470903$ 


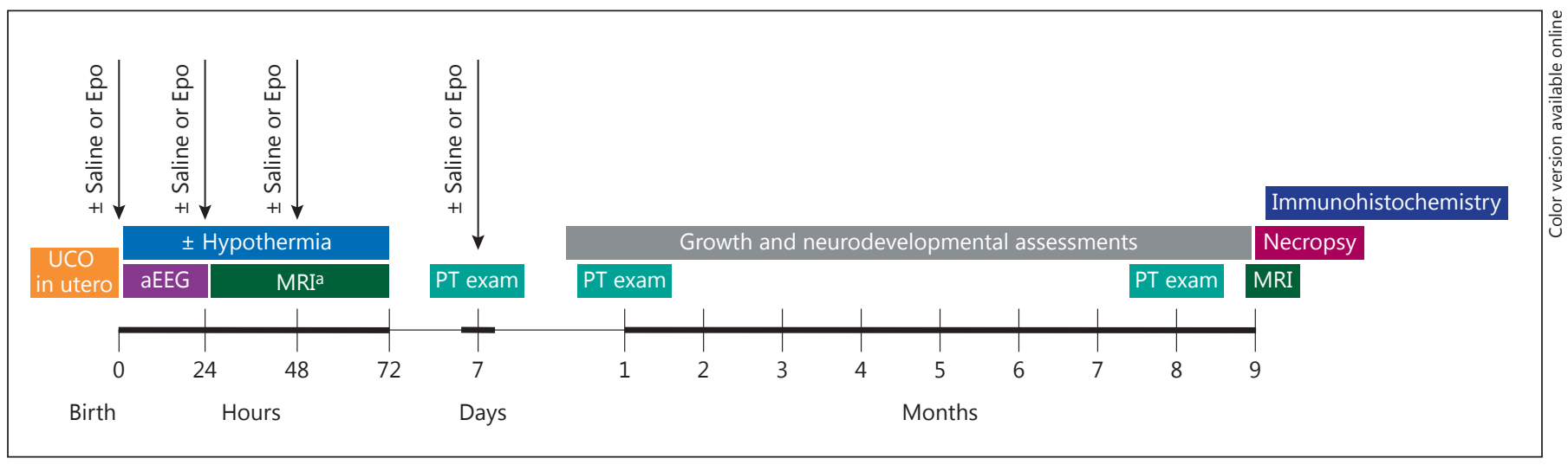

Fig. 1. An overview of the procedures and assessments for the nonhuman primate model of perinatal asphyxia. UCO, umbilical-cord occlusion; Epo, erythropoietin; aEEG, amplitude-integrated elec- troencephalography; MRI, magnetic resonance imaging (and diffusion tensor imaging); PT, physical therapy. ${ }^{\text {a }}$ The first MRI scan was performed at either 24 or $72 \mathrm{~h}$ of age. rane. Following uterine incision, the umbilical cord was exteriorized (while keeping the amniotic fluid and the fetus in the uterine cavity) and clamped (UCO group; $n=25)$ for either $15(n=9)$ or $18 \mathrm{~min}(n=16)$ to induce perinatal asphyxia. Control animals $(n=9)$ were also delivered by hysterotomy, but did not undergo UCO. A 2.5-Fr Vygon ${ }^{\mathrm{TM}}$ umbilical arterial catheter was inserted prior to delivery. Fetuses were delivered, weighed, and stabilized by a team of neonatologists using standardized American Academy of Pediatrics neonatal resuscitation principles [19]. Resuscitations included endotracheal intubation, positive-pressure ventilation, chest compressions, and bolus epinephrine, as indicated. APGAR scores were assigned at 1, 5, 10, and $20 \mathrm{~min}$. A covered heating-pad, radiant warmer, and polyethylene sheet were used to provide initial thermal support during stabilization, followed by placement into a thermal-neutral incubator. Monitoring included pulse oximetry, rectal thermometry, and aEEG (BrainZ BRM3; Natus Medical Incorp., San Carlos, CA, USA).

\section{Treatment Groups}

To focus on the long-term neuropathological effects of perinatal asphyxia, data from the UCO animals were compared with data from control animals. The 25 animals delivered after UCO for either 15 or $18 \mathrm{~min}$ were assigned to 1 of 3 treatment groups: saline $(n=11 ; 3 \mathrm{UCO} \times 15 \mathrm{~min}$ and $8 \mathrm{UCO} \times 18 \mathrm{~min})$, TH only $\left(33.5^{\circ} \mathrm{C}\right.$ for $72 \mathrm{~h} ; n=6,2 \mathrm{UCO} \times 15 \mathrm{~min}$ and $4 \mathrm{UCO} \times 18 \mathrm{~min})$, or $\mathrm{TH}+$ Epo $(n=8,4 \mathrm{UCO} \times 15 \mathrm{~min}$ and $4 \mathrm{UCO} \times 18 \mathrm{~min})$. The Epo dose was $3,500 \mathrm{U} / \mathrm{kg} \times 1$ dose followed by 3 doses of $2,500 \mathrm{U} / \mathrm{kg}$, or Epo $1,000 \mathrm{U} / \mathrm{kg} /$ day $\times 4$ doses given intravenously. Nine animals of similar gestational age were delivered by cesarean section to serve as control animals. Primary outcome measurements were made using the allocated treatment group as the variable. For the secondary analysis, as previously described [18], animals were designated as no-CP (normal or mild CP only), or CP (moderate-to-severe $\mathrm{CP})$.

\section{Animal Care}

Post-resuscitation care, which closely mimicked care practices in human newborns requiring intensive care, was conducted as previously reported [20]. Briefly, study animals were maintained for a minimum of 3 days on parenteral fluids, with adjustments made to maintain euglycemia and hydration. For infants treated with $\mathrm{TH}$, enteral feedings were started after rewarming, once the animals had a normal abdominal exam and stooling was established (typically on postnatal day 4). Routine labs included serial electrolytes, arterial blood gas, and lactate level measurements (iSTAT ${ }^{\circledR}$; HESKA Corp., Loveland, CO, USA). Animals with clinical seizure activity were treated with phenobarbital $(5 \mathrm{mg} / \mathrm{kg})$, with repeated doses given (dose range: $5-30 \mathrm{mg} / \mathrm{kg}$ ) until the seizure activity ceased. Data for all of these parameters have been reported previously $[18,20]$.

Psychological and environmental enrichment activities were performed based on the Infant Primate Research Laboratory Care Protocol [21]. A physical therapist skilled in neonatal care and blinded to the treatment group performed sequential exams at 1 week, 1 month, and 8 months, to document any evidence of motor abnormalities and contractures consistent with CP. Animals were evaluated on their ability to control active movement, and muscle tone at each joint was graded on the Ashford scale of 0 (normal) to 4 (affected parts rigid in flexion or extension) [19]. Based on serial evaluations, animals at the end of the study were characterized as being normal (no CP), or having mild, moderate, or severe CP. Animals who died were placed in the severe CP category.

\section{MRI Acquisition and Analyses}

All surviving UCO animals, non-UCO cesarean section-delivered control animals, and 5 colony animals underwent sedated

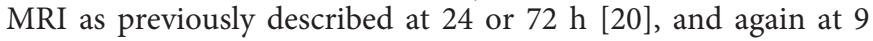
months. Brain MRI from surviving animals diagnosed with CP ( $n=6 ; 1$ severe, 2 moderate, and 3 mild) were compared to control animals $(n=5)$. Total scan time was approximately $2 \mathrm{~h}$ and consisted of magnetization-prepared rapid gradient echo (MPRAGE), high-resolution T1-weighted imaging, DTI, and MR spectroscopy acquired on a Philips Achieva 3.0-tesla magnet with an X-series Quasar dual-gradient system. Two 8-channel-array head coils were custom-made to fit neonatal and juvenile macaques. Details of the sequence acquisition performed were as previously reported [20]. 


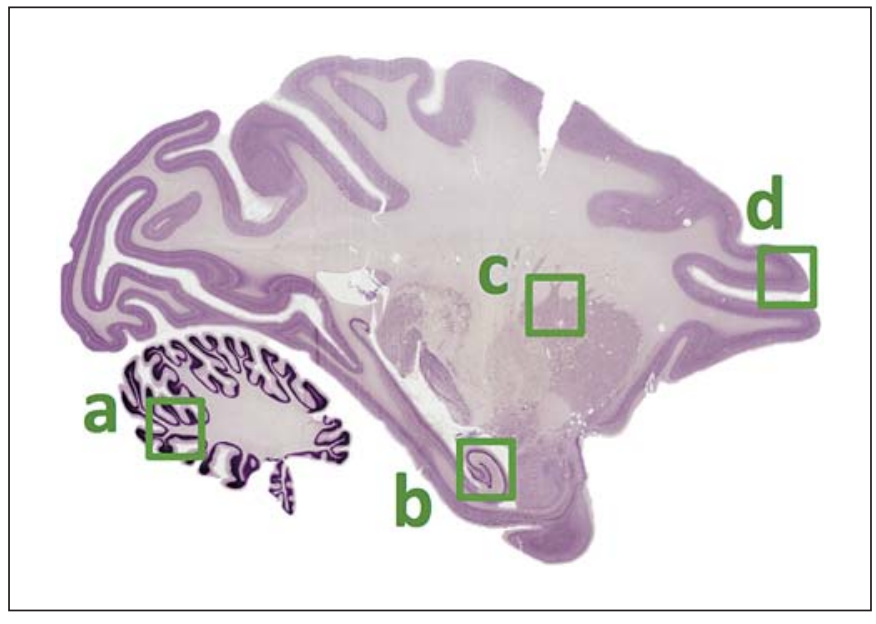

Fig. 2. Representative outline of brain areas analyzed on immunohistochemistry shown on an illustrative sagittal section of macaque brain. H\&E. a, cerebellum in which the white matter and/or granule cell layer were analyzed; $\mathbf{b}$, hippocampus in which cell counts of the whole cornu amnios layer or densitometry with the CA1 central to the images were made; $\mathbf{c}$, corona radiata white-matter tract; $\mathbf{d}$, prefrontal cortex, where analysis was centered on layer 4 .

Table 1. List of antibodies and suppliers

\begin{tabular}{llll}
\hline Antibody & Supplier & Reference & Dilution \\
\hline GFAP & DAKO & Z0334 & $1: 500$ \\
Iba1 & Wako & $019-19741$ & $1: 200$ \\
CD68 & DAKO & m0814 & $1: 500$ \\
CD45 & DAKO & m0701 & $1: 500$ \\
Ki67 & DAKO & M7240 & $1: 500$ \\
MBP & Chemicon & MAB 382 & $1: 500$ \\
Olig2 & IBL & OD803 & $1: 200$ \\
NeuN & Chemicon & MAB377 & $1: 2,000$ \\
Calbindin & SWANT & 300 & $1: 2,000$ \\
MAP2 & Millipore & MAB 378 & $1: 200$ \\
BrdU & BD & 347580 & $1: 100$ \\
Goat anti-rabbit & Vector Labs. & BA-9200 & $1: 500$ \\
Goat anti-mouse & Vector Labs. & BA-1000 & $1: 500$ \\
\hline
\end{tabular}

\section{Diffusion Tensor Imaging}

Voxelwise statistical analysis of the fractional anisotropy (FA) data was carried out using TBSS (Tract-Based Spatial Statistics [22]), part of the FMRIB's Software Library (FSL) [23]. First, FA images were created by fitting a tensor model to the raw diffusion data using FDT, and then brain-extracted using BET [24]. All subjects' FA data were then aligned into a common space using the nonlinear registration tool FNIRT $[25,26]$, which uses a B-spline representation of the registration warp field [27]. Scans at 3 days and at 9 months of age from representative control animals were used to align animals instead of the human normal space. Next, the mean FA image was created and thinned to create a mean FA skeleton, which represents the centers of all tracts common to the group. Each subject's aligned FA data were then projected onto this skeleton, and the resulting data were fed into voxelwise crosssubject statistics. Voxelwise cross-subject statistics were performed on non-FA DTI data (first eigenvalue, second eigenvalue, third eigenvalue, mean diffusivity (MD), mode of anisotropy, volume ratio, and raw T2 signal without diffusion-weighting) using the tbss_non_FA command. Threshold-free cluster enhancement results with correction for multiple comparisons were used to determine significance [28].

\section{Histopathological Analysis}

At 9 months of age, animals were sedated with ketamine and then euthanized with an overdose of intravenous sodium pentobarbital. After terminal perfusion with $4 \%$ paraformaldehyde, the brains were immediately removed, immersion-fixed with $4 \%$ paraformaldehyde, and paraffin-embedded. Hematoxylin and eosin $(\mathrm{H} \& \mathrm{E})$ stains from a subgroup of animals with $\mathrm{CP}(n=3,2 \mathrm{UCO}$ animals and $1 \mathrm{UCO}+\mathrm{TH}$ animal) were compared to the cesareansection control animals $(n=4)$. Sections $(4-\mu$ m-thick $)$ from the level of the thalamus with the associated cerebral cortex, corpus callosum, and hippocampus were routinely processed, embedded in paraffin, and H\&E-stained. These slides were qualitatively evaluated in a blinded manner by a board-certified veterinary pathologist (J.S.).

\section{Immunohistochemical Staining}

Paraffin-embedded sections (6- $\mu \mathrm{m}$-thick) were cut and immunohistochemical (IHC) staining was performed as previously described $[29,30]$, including citric acid antigen retrieval, blocking with $2 \%$ serum (respective to secondary antibody species), followed by incubation with primary (overnight) and secondary (for $1 \mathrm{~h}$ ) antibodies. BrdU staining was performed as previously described including $2 \mathrm{~mol} / \mathrm{L} \mathrm{HCl}$ treatment for $30 \mathrm{~min}$ followed by $0.1 \mathrm{~mol} / \mathrm{L}$ sodium borate for $5 \mathrm{~min}$. The antibodies and concentrations used are listed in Table 1.

Glial fibrillary acidic protein (GFAP), ionized calcium-binding adaptor molecule 1 (Iba1), myelin basic protein (MBP), and microtubule-associated protein 2 (MAP2) underwent densitometric analysis as previously described $[31,32]$ using Image (NIH, USA). Counts were made of CD68, CD45, Ki67, Olig2, NeuN, calbindin and BrdU. Figure 2 demonstrates brain regions evaluated with IHC staining. Analyses were made in 3 fields of view (FOV)/brain region for densitometric analysis and $2 \mathrm{FOV} / \mathrm{brain}$ region for counts as previously described $[31,32]$. IHC data across FOV were averaged and the mean used in a statistical analysis performed with GraphPad Prism v5. IHC analyses of the effects of UCO were made with the Mann-Whitney $U$ test, and the effects of treatment on UCO injury were analyzed using a one-way ANOVA with the Dunnett post hoc test (comparing UCO only to UCO $+\mathrm{TH}$ and to $\mathrm{UCO}+\mathrm{TH}+$ Epo) which included an adjustment for multiple comparisons. Comparisons of $\mathrm{CP}$ versus control for all brain regions were made using the Mann-Whitney $\mathrm{U}$ test. Significance for all analyses was accepted at $p<0.05$.

\section{Brainstem Analysis}

Brainstem tissues were embedded in paraffin and 4- $\mu$ m-thick sections from the pons and medulla were H\&E-stained or immunostained using the Ventana Bench Mark Ultra automated immunostainer (Ventana Medical Systems, Tucson, AZ, USA) according to the manufacturer's instructions. Sections were subjected to 


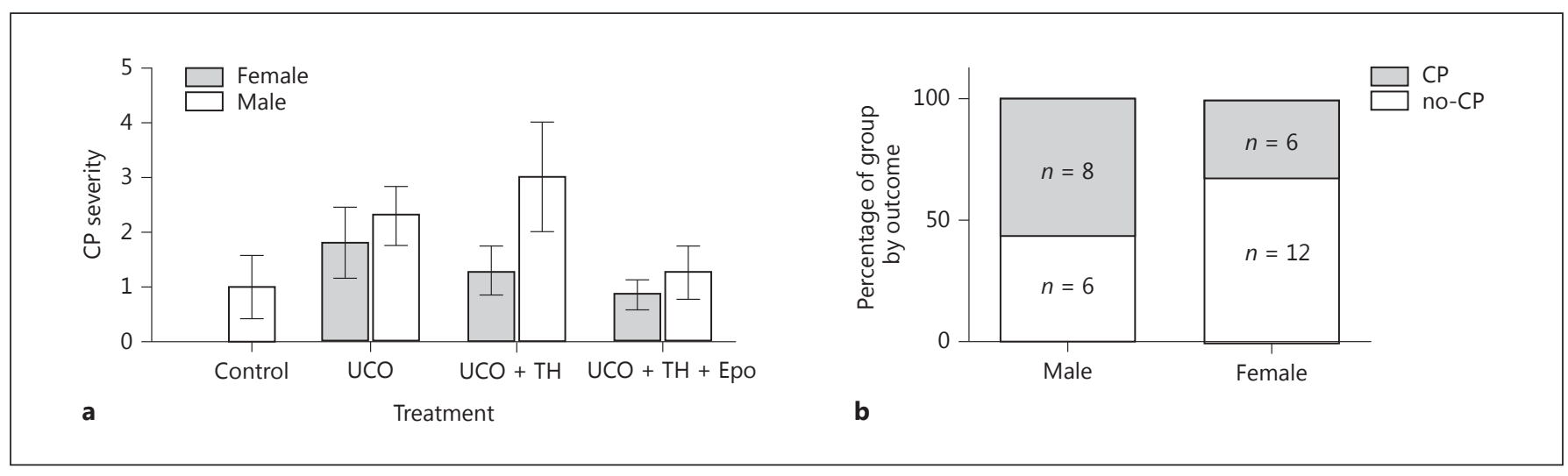

Fig. 3. Sex-specific analysis by treatment and outcome. a Males suffered from more CP $(p=0.029)$ and CP overall was reduced by treatment ( $p=0.021$, two-way ANOVA). $\mathbf{b}$ In an assessment of the proportion of animals suffering from $\mathrm{CP}$, male sex was highly associated with a diagnosis of $\mathrm{CP}(p<0.01$, Fisher exact test).

an antigen retrieval process for 8 min using Ventana's Cell Conditioner 1 (Ventana Medical Systems) followed by GFAP detection using an anti-GFAP antibody (Ventana Medical Systems).

\section{Brainstem Scoring}

Brainstems were analyzed in 12 animals (5 controls, 5 UCO, and $2 \mathrm{UCO}+\mathrm{TH})$. The samples in each case included various levels of medulla, pons, and/or spinal cord. The brainstem was hemisected in most cases. H\&E and GFAP slides from each case were accessed. In assessing H\&E sections, the extent of neuronal loss and the presence or absence of gliosis as defined by the presence of gemistocytic cells with hypereosinophilic cytoplasm (reactive astrocytes) were noted. In assessing GFAP sections, the presence of glial-fiber staining with a "feltwork" appearance in the tissue with and without gliosis as defined by the presence of GFAP-positive astrocytic-cell-body staining (reactive astrocytes) was noted. After assessing the H\&E and GFAP stains, composite scores were given as follows: $0=$ no pathology (H\&E: no neuronal loss and GFAP: a mainly feltwork pattern); $1=$ mild gliosis with no obvious neuronal loss; 2 = moderate gliosis with possible neuronal loss but mild; 3 = severe injury with widespread gliosis and neuronal loss. To assess the strength and direction of association between brainstem severity scores and CP severity scores, the Spearman rank correlation coefficient was used (GraphPad Prism).

\section{Morphometric Measurements of the Cerebellum}

After brain MRI, cerebellar tissue was available for $4 \mathrm{CP}$ and 4 no-CP (control or no UCO) animals for morphometric cerebellar measurements. The cerebellum was embedded in paraffin, and sagittal sections 4 - $\mu \mathrm{m}$-thick were made. Slides were stained for $\mathrm{H} \& \mathrm{E}$, calbindin, GFAP, and BrdU on the Leica Bond Automated Immunostainer with Leica bond kits (Leica Microsystems Inc., Buffalo Grove, IL, USA). Slides were baked for $30 \mathrm{~min}$ at $60^{\circ} \mathrm{C}$ and deparaffinized. Antigen retrieval was performed with citrate/ EDTA buffer for $20 \mathrm{~min}$ at $100^{\circ} \mathrm{C}$. Slides were blocked in normal donkey serum (10\% in TBS) for $10 \mathrm{~min}$ at room temperature. Primary antibodies were applied at room temperature for $30 \mathrm{~min}$. Leica goat anti-mouse HRP polymer was applied for $30 \mathrm{~min}$ at room temperature and blocked with peroxide for $10 \mathrm{~min}$. Leica
Bond Refine Detection (DAB) was applied twice for $10 \mathrm{~min}$ at room temperature. Slides were counterstained with hematoxylin.

Slides were scanned using a Nanozoomer Digital Pathology slide scanner (Olympus America; Center Valley, PA, USA). The digital images were then imported into Visiopharm software (Hørsholm, Denmark) for analysis. Measurements included: thickness of the molecular layer, Purkinje number, Bergman glia density, density of the internal granular layer, density of cells in the white matter, and BrdU+ cell counts. Cerebellar analyses were performed using ImageJ software. Statistical analysis (2-sided $t$ tests; $p<0.05$ ) was performed using IBM SPSS Statistics v9.0 (SPSS Inc., IBM Co., Chicago, IL, USA).

\section{Results}

\section{Animal Characteristics}

After delivery, all animals exposed to UCO were flaccid without spontaneous respirations or movement, required intubation and mechanical ventilation, and met the standard diagnostic criteria for human neonates with moderate-to-severe HIE. These animals had APGAR scores of $<5$ at $10 \mathrm{~min}$ of age, needed positive-pressure ventilation at 10 min of age, had severe metabolic acidosis within the first 60 postnatal min, and had depressed voltages on aEEG. Marginally more females $(19 / 34 ; 55.9 \%)$ than males $(15 / 34 ; 44.1 \%)$ were included in this study. Body weights were not significantly different at necropsy whether assessed by treatment group, outcome (CP), or sex.

\section{Primary Outcome Measurements}

Effect of Treatment on the Diagnosis of CP

Of the 34 animals in this analysis, 4 had moderate-tosevere $\mathrm{CP}$ ( $3 \mathrm{UCO}$ and $1 \mathrm{UCO}+\mathrm{TH}$ ) and 9 had mild $\mathrm{CP}$, 
Table 2. IHC analysis by treatment group

\begin{tabular}{|c|c|c|c|c|c|c|c|}
\hline Target & $\mathrm{Ab}$ & Treatment & Hippocampus & Corona radiata & Cortex & \multicolumn{2}{|c|}{$\begin{array}{l}\text { Cerebellum } \\
\text { (white and grey matter) }\end{array}$} \\
\hline \multirow[t]{4}{*}{ Neurons } & \multirow[t]{4}{*}{ NeuN } & Control & $78.34 \pm 6.01$ & $27.73 \pm 5.19$ & $146.1 \pm 18.14^{*}$ & \multirow{4}{*}{\multicolumn{2}{|c|}{$\begin{array}{l}94.93 \pm 4.94^{* *} \\
63.48 \pm 6.22 \\
82.47 \pm 8.04 \\
73.24 \pm 7.29\end{array}$}} \\
\hline & & UCO & $73.06 \pm 4.52$ & $23.17 \pm 8.54$ & $107.3 \pm 5.92$ & & \\
\hline & & $\mathrm{UCO}+\mathrm{TH}$ & $72.52 \pm 7.43$ & $31.40 \pm 6.07$ & $109.1 \pm 13.93$ & & \\
\hline & & $\mathrm{UCO}+\mathrm{TH}+\mathrm{Epo}$ & $79.06 \pm 4.72$ & $24.57 \pm 4.26$ & $110.5 \pm 9.19$ & & \\
\hline \multirow[t]{4}{*}{ Oligodendrocytes } & \multirow[t]{4}{*}{ Olig2 } & Control & $148.5 \pm 32.50$ & $300.4 \pm 38.02$ & $355.4 \pm 27.77$ & \multirow{4}{*}{\multicolumn{2}{|c|}{$\begin{array}{c}356.40 \pm 42.88^{*} \\
243.5 \pm 27.80 \\
304.0 \pm 27.10 \\
282.4 \pm 37.64\end{array}$}} \\
\hline & & UCO & $118.0 \pm 36.29$ & $246.2 \pm 24.07$ & $352.8 \pm 41.52$ & & \\
\hline & & $\mathrm{UCO}+\mathrm{TH}$ & $213.0 \pm 35.68$ & $242.8 \pm 34.26$ & $320.6 \pm 37.11$ & & \\
\hline & & $\mathrm{UCO}+\mathrm{TH}+\mathrm{Epo}$ & $188.5 \pm 32.50$ & $272.6 \pm 19.95$ & $314.8 \pm 41.30$ & & \\
\hline \multirow[t]{4}{*}{ Myelin } & \multirow[t]{4}{*}{ MBP } & Control & $17.25 \pm 2.49^{*}$ & $61.80 \pm 36.08^{*}$ & $17.07 \pm 2.24$ & \multirow{4}{*}{\multicolumn{2}{|c|}{$\begin{array}{l}69.67 \pm 6.03 \\
58.85 \pm 6.06 \\
68.93 \pm 6.08 \\
64.95 \pm 7.89\end{array}$}} \\
\hline & & UCO & $10.40 \pm 0.99$ & $34.92 \pm 3.38$ & $15.92 \pm 2.42$ & & \\
\hline & & $\mathrm{UCO}+\mathrm{TH}$ & $12.42 \pm 1.22$ & $30.00 \pm 0.87$ & $18.33 \pm 3.15$ & & \\
\hline & & $\mathrm{UCO}+\mathrm{TH}+\mathrm{Epo}$ & $27.53 \pm 3.23^{\# \# \#}$ & $23.81 \pm 2.71^{\#}$ & $16.06 \pm 0.97$ & & \\
\hline \multirow[t]{4}{*}{ Astrocytes } & \multirow[t]{4}{*}{ GFAP } & Control & $39.94 \pm 6.43$ & $55.27 \pm 1.64$ & $31.47 \pm 4.55$ & \multirow{4}{*}{\multicolumn{2}{|c|}{$\begin{array}{l}66.20 \pm 5.86^{* * *} \\
34.70 \pm 2.01 \\
68.86 \pm 3.14^{\S \S \S} \\
54.95 \pm 3.89^{\# \# \#}\end{array}$}} \\
\hline & & $\mathrm{UCO}$ & $33.22 \pm 3.83$ & $50.63 \pm 2.42$ & $29.17 \pm 3.57$ & & \\
\hline & & $\mathrm{UCO}+\mathrm{TH}$ & $39.67 \pm 2.51$ & $58.10 \pm 2.45$ & $28.90 \pm 4.57$ & & \\
\hline & & $\mathrm{UCO}+\mathrm{TH}+\mathrm{Epo}$ & $34.83 \pm 2.12$ & $57.62 \pm 4.24$ & $31.33 \pm 2.52$ & & \\
\hline \multirow[t]{4}{*}{ Microglia } & \multirow[t]{4}{*}{ Ibal } & Control & $25.78 \pm 4.74$ & $18.00 \pm 2.09$ & $20.80 \pm 3.61$ & \multirow{4}{*}{\multicolumn{2}{|c|}{$\begin{array}{r}11.47 \pm 1.58 \\
11.91 \pm 1.38 \\
11.91 \pm 1.38 \\
8.05 \pm 0.77\end{array}$}} \\
\hline & & UCO & $16.61 \pm 2.75$ & $19.52 \pm 0.60$ & $19.33 \pm 1.96$ & & \\
\hline & & $\mathrm{UCO}+\mathrm{TH}$ & $26.83 \pm 3.55$ & $19.71 \pm 0.68$ & $19.67 \pm 0.43$ & & \\
\hline & & $\mathrm{UCO}+\mathrm{TH}+\mathrm{Epo}$ & $19.45 \pm 4.10$ & $18.28 \pm 3.18$ & $20.97 \pm 1.29$ & & \\
\hline \multirow{4}{*}{$\begin{array}{l}\text { Macrophages/ } \\
\text { Microglia }\end{array}$} & \multirow[t]{4}{*}{ CD68 } & Control & $19.09 \pm 2.79 *$ & $15.60 \pm 2.34$ & $12.27 \pm 2.86$ & $20.41 \pm 0.99$ & $5.10 \pm 1.03$ \\
\hline & & UCO & $10.19 \pm 2.96$ & $15.09 \pm 2.69$ & $11.72 \pm 1.25$ & $21.65 \pm 3.08$ & $8.16 \pm 2.13$ \\
\hline & & $\mathrm{UCO}+\mathrm{TH}$ & $14.47 \pm 4.04$ & $22.13 \pm 3.74$ & $10.73 \pm 1.19$ & $33.20 \pm 7.68$ & $6.20 \pm 1.21$ \\
\hline & & $\mathrm{UCO}+\mathrm{TH}+\mathrm{Epo}$ & $16.36 \pm 1.38$ & $12.12 \pm 1.79$ & $13.95 \pm 2.22$ & $20.41 \pm 2.03$ & $4.64 \pm 0.50$ \\
\hline \multirow[t]{4}{*}{ Proliferation } & \multirow[t]{4}{*}{ Ki67 } & Control & $3.00 \pm 0.86$ & $6.87 \pm 2.11$ & $0.33 \pm 0.11^{*}$ & $0.00 \pm 0.00$ & $0.50 \pm 0.50$ \\
\hline & & UCO & $9.91 \pm 5.83$ & $12.26 \pm 2.96$ & $2.15 \pm 0.39$ & $1.79 \pm 0.74$ & $2.67 \pm 1.84$ \\
\hline & & $\mathrm{UCO}+\mathrm{TH}$ & $3.87 \pm 1.08$ & $6.40 \pm 2.07$ & $1.86 \pm 0.34$ & $1.40 \pm 0.60$ & $0.40 \pm 0.40$ \\
\hline & & $\mathrm{UCO}+\mathrm{TH}+\mathrm{Epo}$ & $2.16 \pm 0.65$ & $4.99 \pm 1.76$ & $1.55 \pm 0.32$ & $1.14 \pm 0.60$ & $1.00 \pm 0.65$ \\
\hline $\begin{array}{r}\text { Cerebellar ana } \\
\text { mia; Epo, erythrop } \\
*, * *, * * * \text { Signif } \\
\S, \S \S, \S \S \S \text { Signific } \\
\text { \#,\#\#,\#\# Signific }\end{array}$ & UCO & $\begin{array}{l}\text { cross the white and } \\
\text { us control (Mann-V } \\
\text { s UCO + TH (ANO } \\
\text { SCO + TH + Eno }\end{array}$ & $\begin{array}{l}\text { matter unless st } \\
\text { hey U test). } \\
\text { with the Dunne } \\
\text { OVA with the }\end{array}$ & thoc test). & & & \\
\hline
\end{tabular}

based on serial behavioral assessments by a neonatal physical therapist blinded to the treatment groups. Two female UCO animals died, 1 at 3 days and 1 at 270 days of age. The early death was due to severe neurological compromise. The late death occurred in a $\mathrm{UCO}+\mathrm{TH}$ animal with a history of persistent tachypnea, that unexpectedly collapsed and was noted on autopsy as having had a combination of endo- and myocarditis with cardiac compromise and chronic pulmonary atelectasis. None of the animals treated with $\mathrm{TH}+$ Epo died or had moderateto-severe CP. Analysis with two-way ANOVA demonstrated that males had a greater burden of CP $(53 \%, n=$ 8,4 mild, 3 moderate, and 1 severe) than females ( $26 \%, 6$ mild) across treatment groups ( $p=0.029)$, and this analy-

CP-Associated Neuropathological Changes in a Primate Model of HIE sis also confirmed our previous reports on the efficacy of our treatments to reduce the burden of $\mathrm{CP}(p=0.021$; Fig. 3a).

Effects of UCO and Treatments on Neuropathology

We initially assessed the effects of UCO on the brain and the effects of TH with or without Epo using immunohistochemistry for neurons (NeuN), astrocytes (GFAP), microglia (Iba1), oligodendrocytes (Olig2), microglia/macrophages (CD68 and CD45), and proliferation (Ki67 and BrdU), (Table 2). Significant effects of UCO were: a reduced number of NeuN-positive cells in the cortex (Fig. 4a) and cerebellum, reduced MBP density in the hippocampus and corona radiata, decreased

Dev Neurosci 2017;39:124-140 DOI: $10.1159 / 000470903$ 
Fig. 4. Representative images of immunohistological staining for $\mathrm{NeuN}$ (a) and GFAP (b) in the cerebellum of control animals delivered by cesarean section (Con), asphyxiated animals (UCO), asphyxiated animals treated with therapeutic hypothermia (TH) and asphyxiated animals treated with $\mathrm{TH}+$ erythropoietin (Epo). Scale bar, $70 \mu \mathrm{m}$.

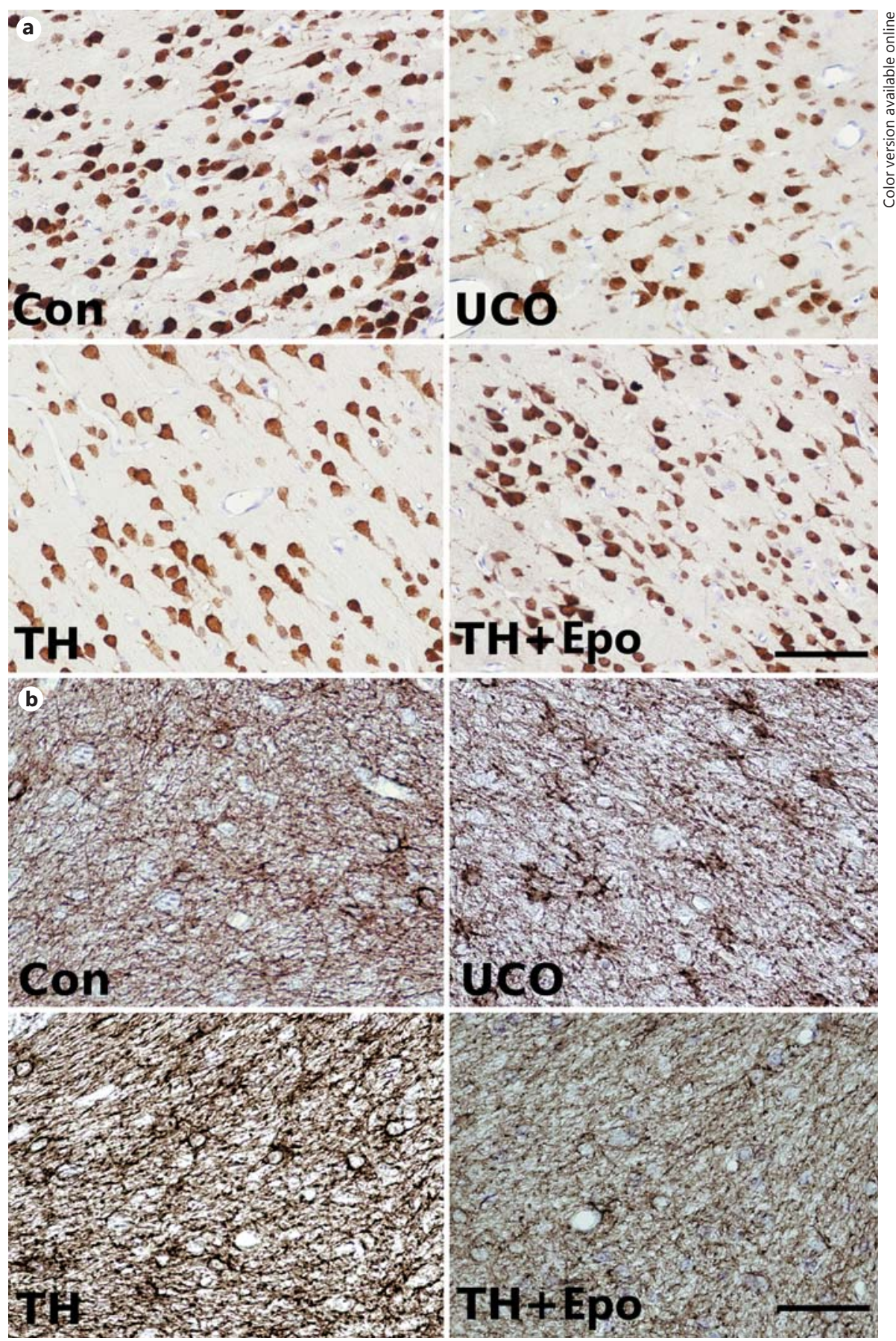

GFAP density in the cerebellum (Fig. 4b), a decreased number of CD68-ositive cells in the hippocampus and an increased number of Ki67-positive cells in the cortex. The effects of $\mathrm{TH}$ or $\mathrm{TH}+$ Epo were minimal, i.e. $\mathrm{TH}+\mathrm{Epo}$ prevented the loss of MBP density in the hippocampus but exacerbated the loss in the corona radiata, and $\mathrm{TH}$ and $\mathrm{TH}+$ Epo prevented the decrease in GFAP staining associated with UCO in the cerebellum. Very sparse staining (1-4 positive cells per entire brain section) was noted for BrdU and so no analysis was conducted. With these measurements, TH + Epo (vs. TH alone) as a therapy was not associated with long-term changes in the brain despite the decrease in the group-wide incidence of $\mathrm{CP}$ (Fig. 3a). The size of the groups precluded any mean- 
Fig. 5. TBSS images show the average fractional anisotropy (FA) image of the subjects, with red areas representing increased signal in control animals versus animals with CP $(p<0.05)$. Scans performed in the first $72 \mathrm{~h}$ demonstrated decreased $\mathrm{FA}$ in the corpus callosum, the anterior and posterior limbs of the internal capsule, and multiple other white-matter tracts in the animals with $\mathrm{CP}$ (6 CP and 4 controls). Blue areas represent regions of increased mean diffusivity (MD) in $\mathrm{CP}$ animals compared to controls $(p<0.05)$. At 9 months, animals with CP continued to have decreased FA in the same areas as found on MRI taken within the first $72 \mathrm{~h}$ after birth (6 CP and 5 controls).

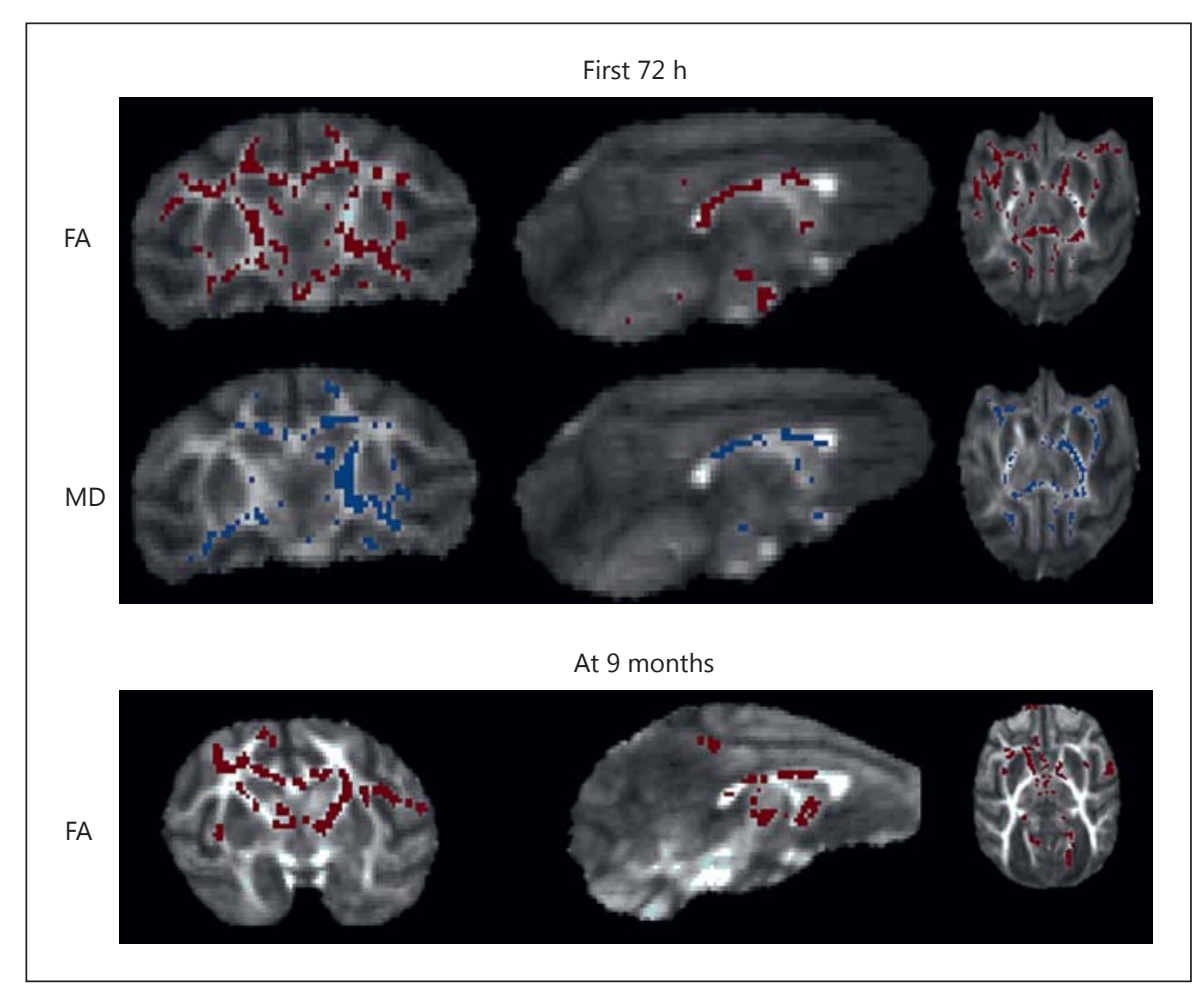

ingful sex-specific analysis of the neuropathology data by treatment group.

\section{Secondary Outcome Measurements}

Effects of Sex on CP Diagnosis

When adjusting our data to proportions of animals suffering from CP in line with our secondary outcomes measurements, we observed a strong association between a diagnosis of CP and male sex (the Fisher exact test, $p<$ 0.0001 ; Fig. 3b). As above, for body weight, no differences by sex were observed for $\mathrm{CP}$ versus no-CP animals.

\section{Changes Associated with CP via MRI}

Fiber density, axonal diameter, and myelination were evaluated by FA measurements. FSL TBSS revealed the changes in FA, the first, second, and third eigenvalues, and the MD of CP and control animals (Fig. 5) in the first $72 \mathrm{~h}$ after injury [33]. Decreased FA was demonstrated in the corpus callosum, the anterior and posterior limbs of the internal capsule, and in multiple other white-matter tracts in the animals with $\mathrm{CP}$ (6 CP and 4 control animals). To examine differences in brain structural integrity between control and $\mathrm{CP}$ animals, MD measurements were obtained; these demonstrated an increased signal in $\mathrm{CP}$ animals compared to in controls. In animals with $\mathrm{CP}$,

CP-Associated Neuropathological Changes in a Primate Model of HIE both the MRI obtained within the first $72 \mathrm{~h}$ of age and the MRI obtained at 9 months of age, demonstrated similar regions of decreased FA (Fig. 5). No differences in brain volume between groups were present.

\section{Neuropathology Associated with CP}

No consistent qualitative abnormalities were observed upon examination of the H\&E-stained slides of the thalamic region of $C P$ animals ( $n=3,1$ mild, 1 moderate, and 1 severe) compared to cesarean-section controls $(n=4,3$ without $\mathrm{CP}$ and 1 mild $\mathrm{CP}$ ). One UCO animal diagnosed with moderate $\mathrm{CP}$ had mild, focally extensive white-matter vacuolation and mild, multifocal expansion of the arteriolar tunica media, of unknown significance (Fig. 6).

In the cortex, compared to the no-CP classified animals, those with CP had decreased numbers of NeuNpositive cells $(p=0.0430)$ but increased numbers of Ki67positive cells $(p=0.001)$ (Fig. 7). In the hippocampus, the numbers of Ki67-positive cells were also increased ( $p=$ 0.0326 ) and CD68-positive cell numbers decreased (vs. animals classified as no-CP) $(p=0.009)$. No other differences in the white matter (corona radiata), cortex, cerebellum, or hippocampus, including staining for Ibal and MBP, were noted. A sex-specific analysis of the neuropathological data revealed that there were no significant

Dev Neurosci 2017;39:124-140 


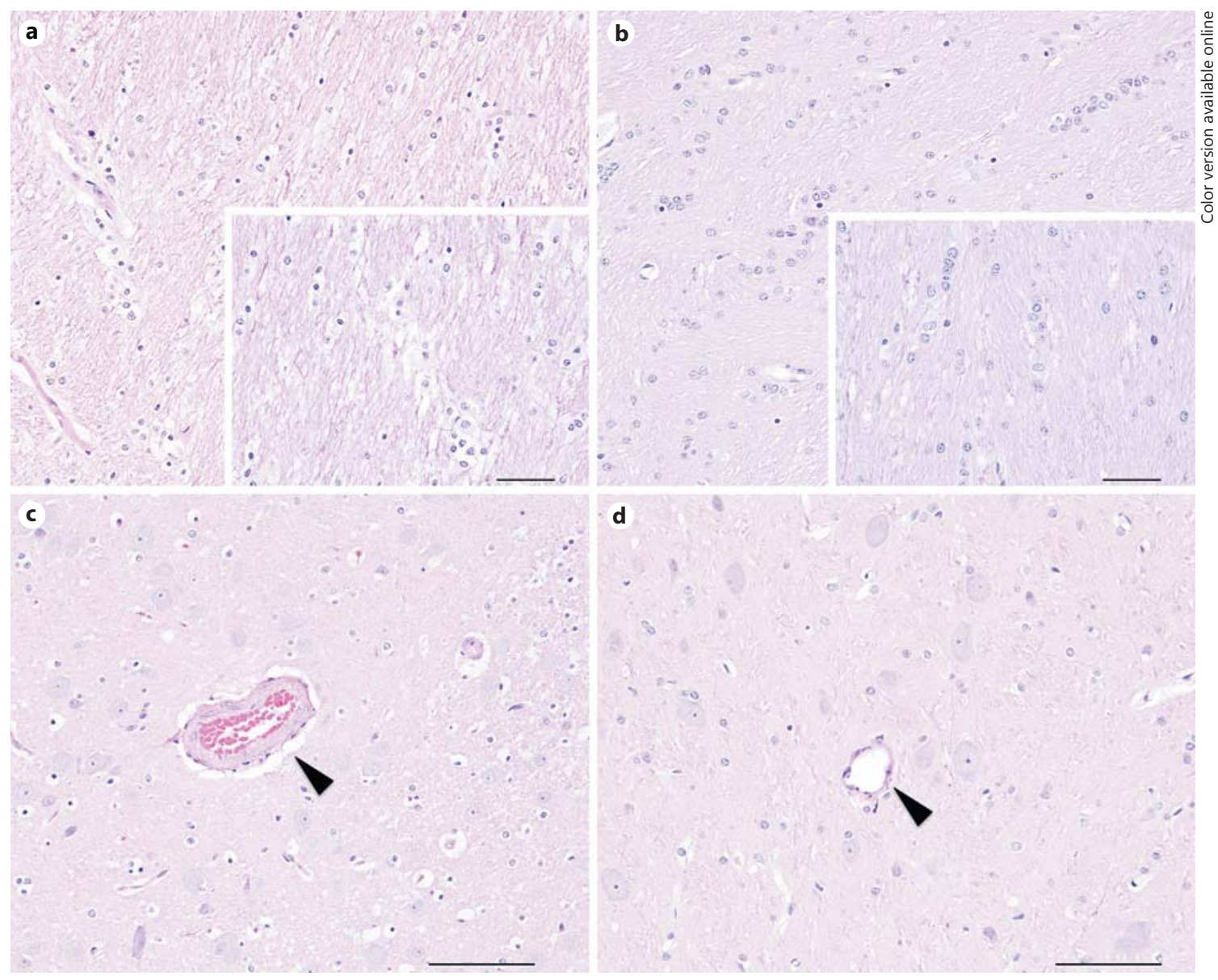

Fig. 6. Images of the brain at the level of the thalamus from 2 animals. a, c Umbilical cord occlusion (UCO) group animal with moderate CP. b, d Control animal. White-matter tracts show mild vacuolation and disorganization (a) compared to the control animal (b). H\&E. $\times 20$. Insets Higher-magnification images (scale bar, $50 \mu \mathrm{m}$ ). c Within the gray matter, the tunica media of the vessel wall (arrowhead) is mildly expanded in the UCO animal. d Representative vessel (arrowhead) from the control animal. Scale bars, $100 \mu \mathrm{m}$.

differences between the CP group and the "no-CP" group in females (online suppl. Fig. 1; for all online suppl. material, see www.karger.com/doi/10.1159/000470903). Analysis specifically of the males revealed that the effects from the whole group analysis remained for neurons in the cortex $(p=0.0879)$, and additionally in the cerebellum $(p=$ 0.0173; online suppl. Fig. 2). Furthermore, in male CP animals, the increase in numbers of Ki67-positive cells in the cortex $(p=0.120)$ and the decrease in numbers of CD68-positive cells in the hippocampus $(p=0.0159)$ were more significant than in the mixed sex analysis. However, the increase in hippocampal Ki67 expression was not significant $(p=0.662)$.
Changes in the Brainstem Associated with CP:

\section{Brainstem Scoring}

Brainstems were analyzed in 12 animals ( 5 controls, 5 $\mathrm{UCO}$, and $2 \mathrm{UCO}+\mathrm{TH}$ ). Abnormalities were noted in all 5 animals with $\mathrm{CP}$ ( $3 \mathrm{UCO}$ and $2 \mathrm{UCO}+\mathrm{TH})$, which included 1 UCO animal that died at 270 days of age. One control animal without CP had a brainstem score of 1 (mild gliosis with no obvious neuronal loss). Animals with $\mathrm{CP}$ demonstrated neuronal loss and reactive astrocytes with increased cytoplasmic GFAP-positive staining compared to the control animals (Fig. 8g). A strong association between brainstem severity scores and CP severity scores was demonstrated by the Spearman rank correlation coefficient $(R=0.973$; Fig. $8 \mathrm{~h})$. 


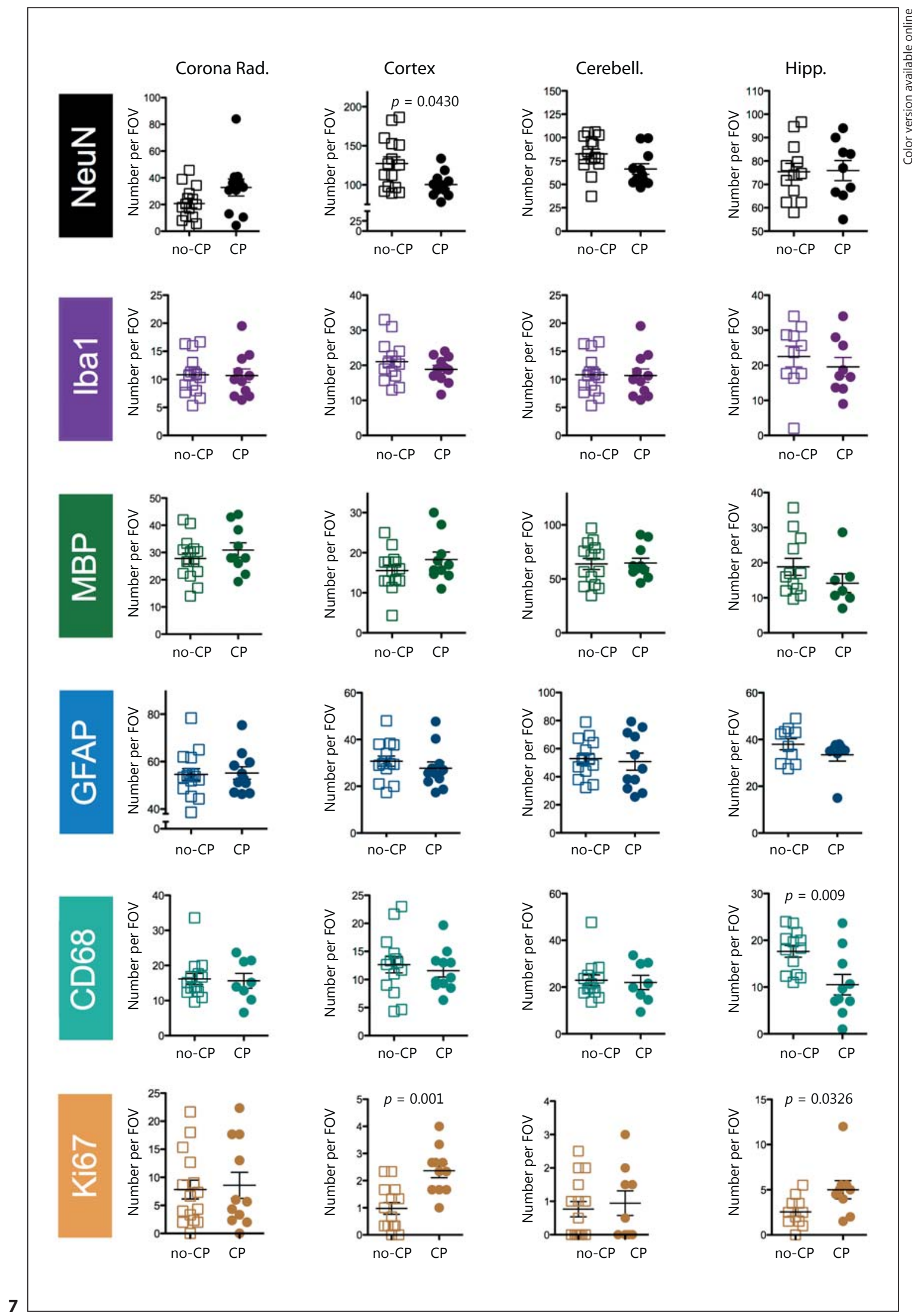

(For legend see next page.) 

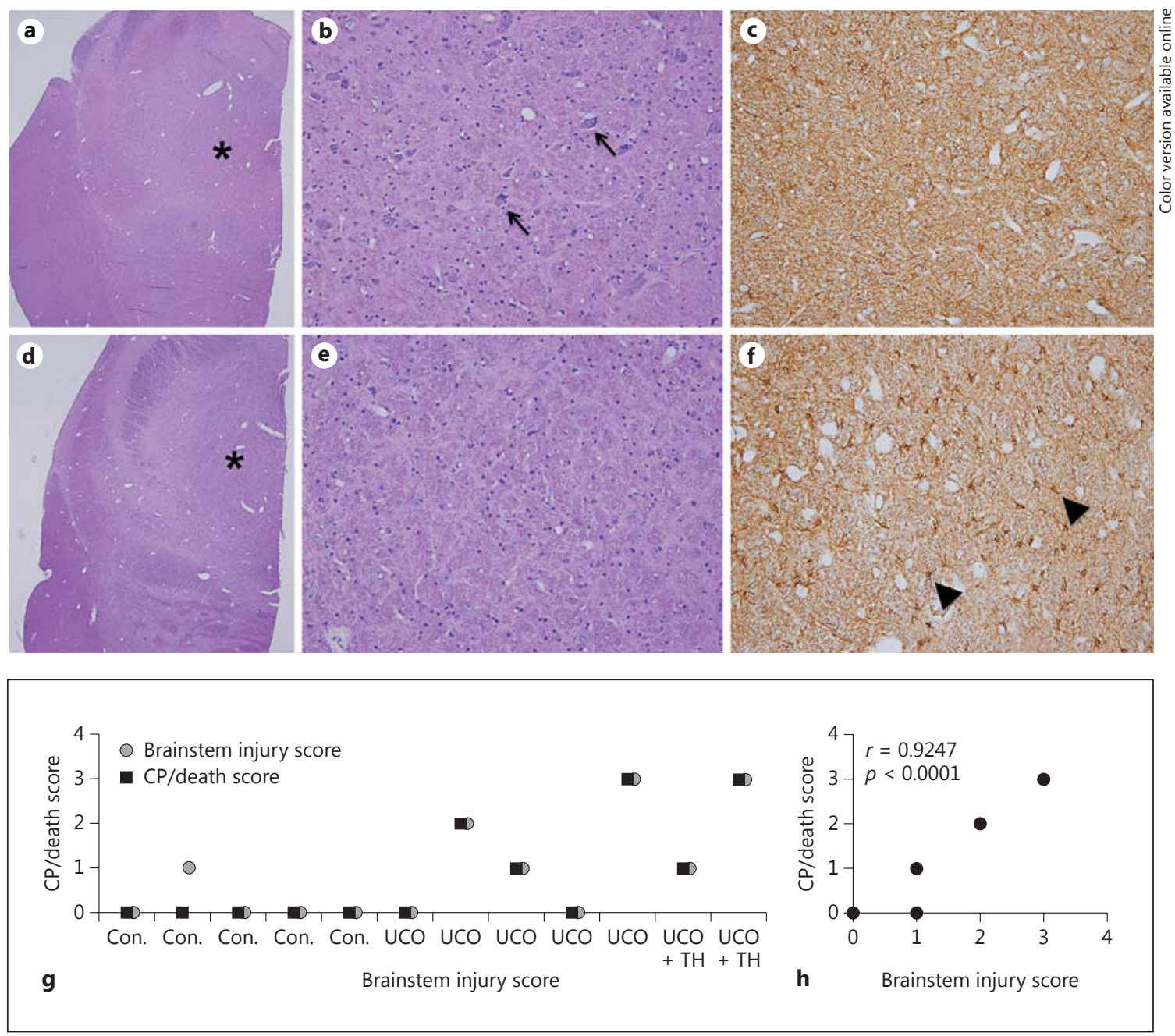

Fig. 8. Histology. a-c Brainstem of a control animal taken at the level of the pons. d-f Brainstem of a CP animal taken at the level of the pons. $\mathbf{a}, \mathbf{d} H \& \mathrm{E} . \times 2$. Asterisks indicate the area of the pontine tegmentum from which images $\mathbf{b}$ and $\mathbf{e}(\mathrm{H} \& \mathrm{E} . \times 20)$ and $\mathbf{c}$ and $\mathbf{f}$ (GFAP. $\times 20$ ) were taken. The CP animal shows neuronal loss compared to the no-CP non-UCO animal ( $b$, examples of neurons are marked with arrows). c The GFAP staining in the control animal shows the normal appearance of astrocytes with predominate staining in the fibers (feltwork pattern) with scattered detectable cell bodies. $\mathbf{f}$ The GFAP staining in the CP animal shows a substantial increase in reactive astrocytes with increased cytoplasmic GFAP-positive staining (examples of reactive astrocytes are

Fig. 7. Scatter plots of the data from the IHC staining separated by outcome. Data are from animals diagnosed with no CP or mild CP ("no-CP") versus animals diagnosed with moderate or severe CP (CP) for the corona radiata (Corona Rad.), cortex, cerebellum marked with arrowheads). $\mathbf{g}$ Relationship between brainstem and $\mathrm{CP} /$ death scores. Brainstem histology severity scores ( $y$ axis) were assessed using $\mathrm{H} \& \mathrm{E}$ and GFAP stains with composite scores given as follows: 0 , no pathology (no neuronal loss [H\&E]; a mainly feltwork pattern [GFAP]); 1, mild gliosis with no obvious neuronal loss; 2, moderate gliosis with possible neuronal loss but mild; 3 , severe injury with widespread gliosis and neuronal loss. CP severity scores ( $x$ axis) were characterized as: 1 , normal (no CP); 2 , mild; 3 , moderate; 4 , severe CP or death. $\mathbf{h}$ Correlation of brainstem and $\mathrm{CP} /$ death scores. Analysis of the brain stem and CP severity scores was demonstrated by the Spearman rank correlation coefficient $(r=0.973)$.

(Cerebell.), and hippocampus (Hipp.) outlined in Figure 2. Groups were compared with the Mann-Whitney $\mathrm{U}$ test and $p$ values $<0.05$ are indicated on the graphs. 


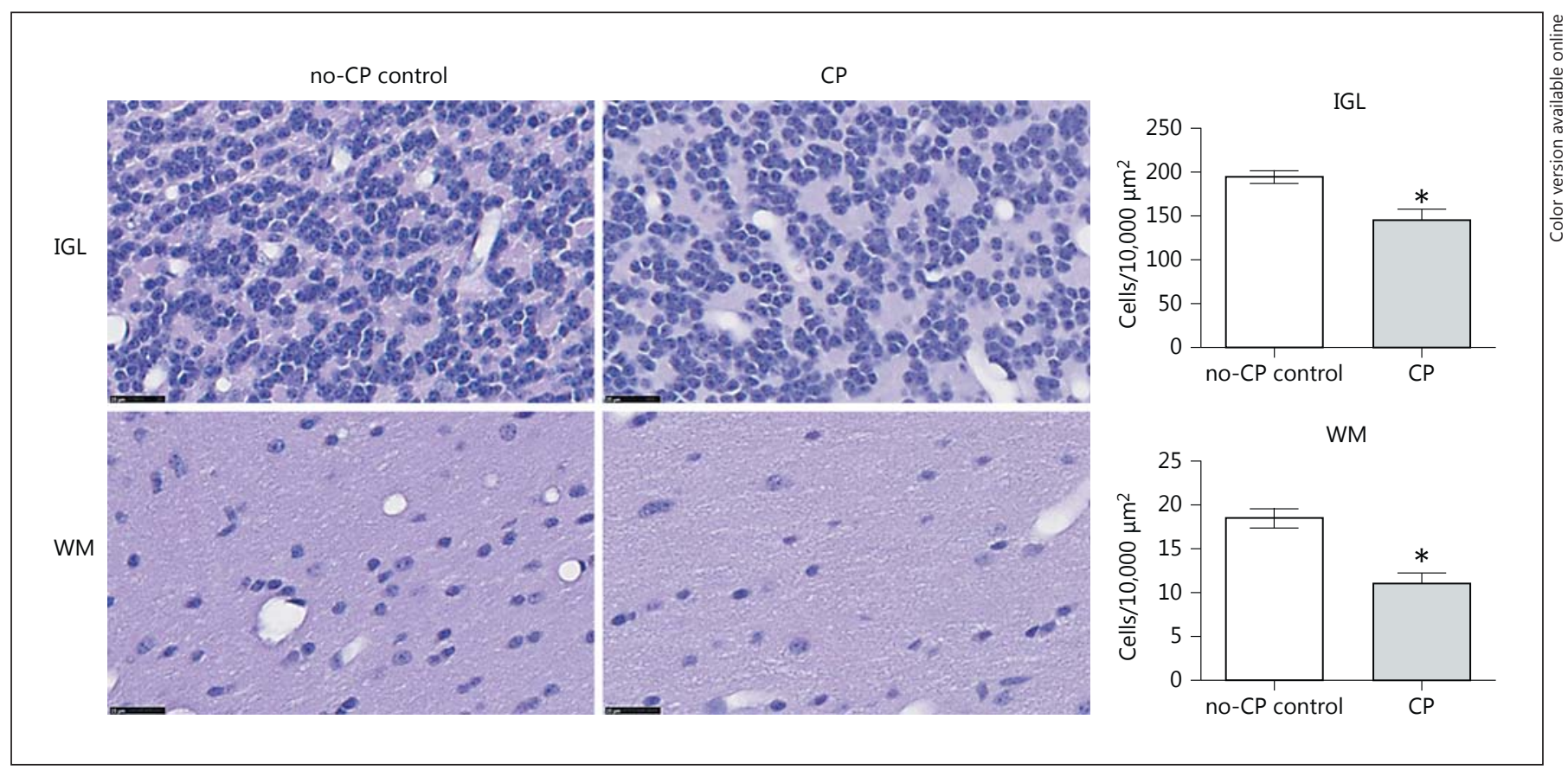

Fig. 9. The cell density in the cerebellar internal granular layer (IGL) and white-matter (WM) tract was compared in controls $(n=5)$ and animals that had CP $(n=4)$. CP animals had decreased cell density in the IGL and WM compared to control animals. ${ }^{*} p<0.05,2$-sided $t$ test. Scale bars, $25 \mu \mathrm{m}$.

Morphometric Measurements of the Cerebellum Associated with CP

Cerebellar tissue was available from $4 \mathrm{CP}$ animals and 4 animals without CP (no-CP; no-UCO control only). At 9 months of age, the external granular layer was not present. There were no differences in thickness of the molecular layer (CP $334 \pm 56$ vs. no-CP controls $263 \pm 26 \mu \mathrm{m}$ ), Purkinje cell density (CP $6.3 \pm 0.8$ vs. no-CP control 6.8 \pm 1.0 cells $/ 500 \mu \mathrm{m}$ ), or Bergman glial-fiber density (CP $20.2 \pm 1.5$ vs. no-CP control $18.2 \pm 2.6$ fibers $/ 100 \mu \mathrm{m}$ ). The cell density of the internal granular layer and white matter was significantly decreased in CP animals (2-sided $t$ tests, $p<0.05$; Fig. 9). No differences in cerebellar volume were evident between groups.

\section{Discussion}

The nonhuman primate brain has a complexity and development similar to the human brain. This facilitates neurocognitive testing to be conducted over time that is analogous to testing done in humans [21]. These attributes make this UCO model attractive for studying the long-term neuropathology of $\mathrm{CP}$ related to $\mathrm{HIE}$ and the

CP-Associated Neuropathological

Changes in a Primate Model of HIE safety and efficacy of neurotherapeutics. We previously reported that, compared to UCO saline, for animals treated with $\mathrm{TH}+$ Epo, there were fewer deaths and $\mathrm{CP}$ at 9 months of age, with a relative risk reduction of 0.911 (95\% CI -0.429 to 0.994$)$ and an absolute risk reduction of 0.395 (95\% CI 0.072-0.635) [18]. The primary purpose of this study was to confirm the long-term safety and efficacy of $\mathrm{TH}+$ Epo, including a neuropathological assessment. We found that $\mathrm{TH}+$ Epo did indeed reduce the long-term risk of $\mathrm{CP}$ or death, and that animals treated with TH + Epo (vs. TH only) were not negatively impacted (e.g., with their somatic growth, head circumference, and neuropathology scores). Based on IHC analysis, there were no signs of long-term toxicity with $\mathrm{TH}+\mathrm{Epo}$, which supports further research of this combined treatment strategy to promote improved long-term outcomes in neonates with HIE.

Our secondary aim was to assess the neuropathology of animals that developed CP by 9 months of age, which is comparable to 3 years of human development, after exposure to UCO compared to animals without CP (i.e., control animals \pm UCO animals with only mild CP). Animals with $\mathrm{CP}$ demonstrated decreased FA and increased $M D$ values across multiple white-matter tracts, including

Dev Neurosci 2017;39:124-140 DOI: $10.1159 / 000470903$ 
the corpus callosum and internal capsule on MRI. In children with congenital hemiparesis and sensorimotor deficits, reduced FA and increased MD have been observed for precentral gyrus and paracentral lobule connections within the posterior limb of the internal capsule on the hemisphere contralateral to the impaired side [34]. The significance of the decreased FA in the anterior limb of the internal capsule, a region which contains thalamocortical corticopontine and caudate/pallidum fibers, is not clear. Bilateral disruptions in the thalamocortical connections of the anterior limb of the internal capsule have been demonstrated in schizophrenia and have been associated with poor memory performance [35]. Decreased whitematter FA is observed in infants diagnosed with HIE and the severity of the loss in FA is associated with the longterm outcome [36].

In neonates with HIE, there is no established correlation of DTI with brain pathology findings; this prevents us from drawing significant conclusions regarding radiological-pathological correlations in our animals with CP. We did not observe changes in either oligodendrocyte cell number (Olig2-positive cell counts, data not shown) or myelin-staining intensity to directly support the notion that decreased FA might relate to hypomyelination. However, important ultrastructural changes such as altered myelin-wrapping efficiency or altered G ratios (axon diameter/total fiber diameter) can affect FA values and are associated with perinatal brain injury $[29,37]$. Gliosis is considered an important contributor to perinatal injury following hypoxia-ischemia $[38,39]$ and it is thought that gliosis might persist, preventing repair and precipitating further neurodegeneration [40]. However, there were no changes in the density of staining for astrocytes (GFAP) or the number of microglia (Iba1+). Given the potential relevance of activation state rather than cell morphology or number in an in vivo context, our analysis might not have had the sensitivity to detect long-term (tertiary) changes in the glia. With regard to the decreased macrophage/microglia number (CD68) in the hippocampus, in isolation, the relevance of this change is purely speculative, e.g. it possibly mediates effects on memory by changing the local inflammatory milieu [41]. Similarly, there appeared to be a long-term, but isolated effect of increased proliferation in the $\mathrm{CP}$ group. This occurred in the cortex, which suffered a reduction in mature neuron numbers; it is tempting to suggest this simply reflects a long-term (failed) regenerative response, but this requires additional work to specifically characterize the type of cells that proliferate.

Animals with CP had decreased staining for cortical neurons and increased brainstem glial scarring compared to animals in the control group. We previously reported that UCO in this model was associated with reduced cerebellar volumes and that this was corrected with $\mathrm{TH}+$ Epo [18]. Providing neuropathological evidence to support our previous work, the cerebellar cell density of the internal granular layer and white matter was decreased in $\mathrm{CP}$ animals compared to in control animals.

There was a strong correlation between brainstem injury severity (neuronal loss and glial scarring) and CP severity. A possible explanation for this neuronal loss could be damaged neural pathways (e.g., motor cortex to brainstem) that led to the demise of remote brainstem neurons following UCO-induced HIE. In a neonatal rat model, Reinebrant et al. [42] demonstrated that disrupted serotonergic inputs to the motor cortex resulted in brainstem serotonergic neuronal loss after hypoxic-ischemic injury. Disruption of the brainstem and spinal cord serotonergic system has also been proposed to account for the motor defects observed in neonatal mice using the Rice-Vanucci hypoxic-ischemic injury model [43]. Future studies on our UCO model, investigating damaged neural pathways, such as the serotonergic system pathway, after neonatal HIE may reveal cellular mechanisms of axonal degeneration relevant to the development of CP.

An important observation from this study was the greater likelihood of males to suffer from CP following exposure to UCO. This observation is in agreement with clinical findings, including by the Surveillance of Cerebral Palsy in Europe (SCPE), that males are more likely to suffer CP following perinatal events such as HIE [44, 45]. We noted (despite the low power in this analysis) that effects specific to males drove the majority of the neuropathological changes in animals suffering CP in this model. Sex-specific differences in basic cellular responses to insult may underpin some of the vulnerability to perinatal events [46], as may differences in the maturation of various regions altering susceptibility [47]. Unfortunately, only the overall outcome and IHC analysis were sufficiently powered to approach the question of sex-specific effects.

There is limited information available on the longterm neuropathology of HIE-related brain injury in relation to cognitive impairments and $\mathrm{CP}$ for guiding strategies to improve childhood outcomes. The inability to accurately determine the cause of HIE in human newborns remains a substantial shortcoming of clinical medicine. Not only are the timing and mechanism of brain injury based on speculation, but the prevention of HIE has been unattainable. Retrospective analyses of HIE in human newborns is typically hindered by the conjecture about
136

Dev Neurosci 2017;39:124-140 DOI: $10.1159 / 000470903$
McAdams/Fleiss/Traudt/Schwendimann/ Snyder/Haynes/Natarajan/Gressens/Juul 
underlying causation, the lack of any accurate information on the duration and degree of insult(s), and nebulous explanations of key factors that predict, trigger, and perpetuate the pathological processes related to HIE. While an imperfect model, the nonhuman primate UCO model of HIE has a clearly defined injury mechanism (UCO) and duration of initial insult (15 or $18 \mathrm{~min}$ of UCO), which allows for the study of treatments initiated at uniform times across groups. Similar to humans, not all animals with HIE developed CP or had apparent long-term neurodevelopment deficits. Why some animals and human newborns tolerate the severe hypoxic insults associated with HIE and others die or are permanently devastated remains a conundrum. While most $\mathrm{CP}$ cases are not secondary to HIE, the cases that are related have been associated with severe HIE based on the Sarnat criteria [48]. Consistent with this relationship, the animals subjected to UCO in our study demonstrated findings consistent with severe HIE, and went on to develop CP in $35 \%(12 / 34)$ cases. In this study [48], TH did not reduce the long-term outcome of death or CP. Whether this discrepancy with human studies is due to the UCO model mechanism of injury, the limited sample size, or other unrecognized factors remains unclear. One factor that must be considered is the duration of cooling, since $72 \mathrm{~h}$ in a neonatal macaque is not equivalent to $72 \mathrm{~h}$ in a human neonate, given that the rate of aging in these animals is approximately 3 times as fast as in humans [49]. A recent phase III trial, studying the effect of the depth and duration of cooling on outcomes of moderate-to-severe HIE, demonstrated a higher adjusted risk ratio for NICU deaths of babies cooled for $120 \mathrm{~h}$ compared to $72 \mathrm{~h}$ (1.37; 95\% CI 0.92-2.04) [50]. Thus, the 72-h duration of TH in the $\mathrm{UCO}+\mathrm{TH}$ animals may have exceeded the window of benefit. While combined treatment with $\mathrm{TH}+$ Epo looks promising $[15,16]$, we suspect that additional strategies will be needed to mitigate long-term neurodevelopmental injury in neonates with moderate-to-severe HIE.

Neonates with HIE typically demonstrate 2 main brain MRI injury patterns, a watershed-predominant or a basal ganglia/thalamus pattern, as demonstrated by Miller et al. [51] who studied a cohort of 173 term neonates with HIE in order to determine the antenatal and perinatal risk factors and neurodevelopmental deficits associated with these patterns. In their study, the newborns delivered by emergency cesarean section who required intensive resuscitation at birth and had more severe encephalopathy and seizures, had a basal ganglia/thalamus injury pattern detected on MRI that was associated with the most impaired motor and cognitive outcome at 30 months. In our

CP-Associated Neuropathological Changes in a Primate Model of HIE nonhuman primate study, UCO induced an acute, abrupt hypoxic insult associated with intensive resuscitation at birth and severe encephalopathy and seizures; however, no pattern of gross brain MRI abnormalities was detected either early (on days 1-3) or later (at 6 or 9 months) [18]. Although these animals did not demonstrate a basal ganglia/thalamus injury pattern typically associated with severe neuromotor and cognitive outcomes in human neonates, 35\% (12/34) still developed CP (4 animals had moderate-to-severe CP and 9 had mild CP). The explanation for this apparent discrepancy between neuroimaging and clinical presentation is not clear. While many children with CP have abnormalities detectable on MRI, this is not always the case, as children with all clinical subtypes of $\mathrm{CP}$, including severe functional severity, can have normal conventional MRI [52]. A retrospective study by $\mathrm{Nu}-$ mata et al. [53], investigating associations between neuroimaging findings, motor function, epileptic episodes, and cognitive function in $86 \mathrm{CP}$ patients with spastic diplegia who were born at term, found no abnormalities on conventional MRI in $41.9 \%$ of the patients. Using a population-based CP registry (Quebec, Canada), Benini et al. [54] studied 213 patients to identify distinctive clinical features characterizing children with CP and normal-appearing MRI findings. They demonstrated that $42 \%$ (15/36) of the children with normal-appearing MRI displayed a high degree of functional disability (Gross Motor Functional Classification System IV-V) versus 33\% (30/90) with abnormal MRI, and that normal-appearing MRI was more common in children with dyskinetic CP and less common in children with spastic hemiplegic $\mathrm{CP}$, compared with other $\mathrm{CP}$ variants. Abnormal connectivity may be important in the development of CP [55]; unfortunately, we did not conduct MRI structural connectivity measurements to determine if animals with CP had disrupted sensorimotor pathways compared to animals without CP.

This study has many limitations, including the small number of animals in each group due to the substantial costs of the model, the differences in UCO durations (15 min in 9 animals and $18 \mathrm{~min}$ in 18 animals), and that neuropathological analysis of only 34/56 animals (60.7\%) from the parent study (only the animals that survived to 9 months were examined) was conducted. This last point is important as it means that we may not have detected the most severe neuropathological injuries as these lead to early death. During the course of the study, the UCO duration was changed from 15 to $18 \mathrm{~min}$ to promote more consistent and significant long-term neurodevelopmental disability, important for testing neuroprotective

Dev Neurosci 2017;39:124-140 DOI: $10.1159 / 000470903$ 
strategies. However, the distribution of animals exposed to UCO for 15 or 18 min was consistent between the groups and across sexes. Since all animals received serial behavioral testing and physical therapy, the long-term neurodevelopmental outcomes may have been modified by this therapy, a phenomenon that could affect the interpretation of the long-term impact of early treatment $(\mathrm{TH}$ \pm Epo) versus serial physical therapy for newborns with HIE. This issue of an apparent enriched environment is important, as physical therapy has been found to improve outcomes across preclinical models [56, 57], and a varied home environment is standard for babies. As such, any therapy must be able to be effective in the context of an enriched environment. Another criticism of our study is that we included animals with mild CP in the "no-CP" group when we analyzed scatter plot data from IHC staining based on outcomes. Due to limited animal numbers, this no-CP group was compared to animals diagnosed with moderate or severe $\mathrm{CP}$ (CP group). We recognize that humans with mild CP can have significant disabilities, including cognitive impairment, behavioral problems, and epilepsy, so future, larger studies would benefit from not combining different categories of $\mathrm{CP}$ in their analysis.

Another study limitation is the lack of IHC staining on thalamic tissue. Thalamic abnormalities were not seen on MRI or with H\&E staining (except for mild changes in 1 animal with moderate $\mathrm{CP}$ ). These findings likely exclude severe/frank thalamic lesions from being involved in the observed outcomes, but, as IHC staining was not performed, we cannot exclude subtle deep gray matter pathology. Brainstem histopathological analysis was limited to 12 animals and did not include any $\mathrm{TH}+$ Epo animals ( $n=8,5$ without $\mathrm{CP}$ and 3 with mild $\mathrm{CP}$ ), which meant assessment of this treatment effect was not possible. In addition, MRI analysis of the brainstem was not performed, so no correlation of radiological and histopathology findings was possible. Given the need for a robust model of HIE, our study was important but it was limited in statistical power due to the ethical and practical limitations of a primate model. This was because multiple regions and cell types were analyzed, which increased the chance of false positive results. However, our neuropathological assessments supplemented strong observations on behavioral deficits, with robust clinical applicability. Therefore, despite its limitations, the study offers valuable insight into the long-term brain neuropathology associated with $\mathrm{CP}$ secondary to HIE, findings that are not ascertainable in humans for obvious ethical reasons and secondary to the limitations of postmortem human studies (including the variability in postmortem delay and in underlying etiologies).

In conclusion, animals with $\mathrm{CP}$ associated with severe HIE secondary to UCO demonstrated decreased FA of multiple white-matter tracts including the corpus callosum and internal capsule, decreased IHC staining for cortical neurons, and increased brainstem glial scarring compared to animals without $\mathrm{CP}$. In addition, the cerebellar cell density of the internal granular layer and white matter was decreased in CP animals compared to in control animals without CP. However, our UCO model was not associated with a basal ganglia/thalamus injury pattern typical of neonates with HIE who develop $\mathrm{CP}$, which suggests possible structural connectivity abnormalities. Future studies using this model should include MRI brainstem imaging, including structural connectivity measurements, to evaluate for disrupted sensorimotor pathways that may play a key role in animals with CP.

\section{Acknowledgments}

We thank Kylie Cory and Tom Gerbert for their animal care work, and Olivia Janson and Henry Smelser for technical assistance.

The study was supported by grants from the NIH/Eunice Kennedy Shriver National Institute of Child Health and Human Development: R01-HD-52820, and HD083091; Inserm, Université Paris Diderot, Université Sorbonne-Paris-Cité, DHU PROTECT, PremUP, Fondation de France, Fondation pour la Recherche sur le Cerveau, Roger de Spoelberch Foundation, Leducq Foundation, and the Wellcome Trust (WSCR P32674). We also acknowledge financial support from the Department of Health via the National Institute for Health Research (NIHR) Comprehensive Biomedical Research Centre Award to Guy's \& St Thomas' NHS Foundation Trust in partnership with King's College London and King's College Hospital NHS Foundation Trust. The supporting bodies played no role in any aspect of study design, analysis, interpretation or decision to publish this data.

References

1 GBD 2013 Mortality and Causes of Death Collaborators: Global, regional, and national age-sex specific all-cause and cause-specific mortality for 240 causes of death, 1990-2013: a systematic analysis for the Global Burden of Disease Study 2013. Lancet 2015;385:117171.

2 Kurinczuk JJ, White-Koning M, Badawi N: Epidemiology of neonatal encephalopathy and hypoxic-ischaemic encephalopathy. Early Hum Dev 2010;86:329-338. $\begin{array}{ll} & \text { Dev Neurosci } 2017 ; 39: 124-140 \\ & \text { DOI: } 10.1159 / 000470903\end{array}$
McAdams/Fleiss/Traudt/Schwendimann/ Snyder/Haynes/Natarajan/Gressens/Juul 
3 Locatelli A, Incerti M, Ghidini A, Greco M, Villa E, Paterlini G: Factors associated with umbilical artery acidemia in term infants with low Apgar scores at $5 \mathrm{~min}$. Eur J Obstet Gynecol Reprod Biol 2008;139:146-150.

4 Shankaran S, Laptook AR, Ehrenkranz RA, Tyson JE, McDonald SA, et al; National Institute of Child Health; Human Development Neonatal Research Network: Whole-body hypothermia for neonates with hypoxic-ischemic encephalopathy. N Engl J Med 2005;353: 1574-1584.

5 Gluckman PD, Wyatt JS, Azzopardi D, Ballard R, Edwards AD, Ferriero DM, Polin RA, Robertson CM, Thoresen M, Whitelaw A, Gunn AJ: Selective head cooling with mild systemic hypothermia after neonatal encephalopathy: multicentre randomised trial. Lancet 2005;365:663-670.

6 Azzopardi DV, Strohm B, Edwards AD, Dyet L, Halliday HL, Juszczak E, Kapellou O, Levene $M$, Marlow N, Porter E, Thoresen M, Whitelaw A, Brocklehurst P; TOBY Study Group: Moderate hypothermia to treat perinatal asphyxial encephalopathy. $\mathrm{N}$ Engl J Med 2009;361:1349-1358

7 Jacobs SE, Morley CJ, Inder TE, Stewart MJ, Smith KR, McNamara PJ, Wright IM, Kirpalani HM, Darlow BA, Doyle LW; Infant Cooling Evaluation Collaboration: Wholebody hypothermia for term and near-term newborns with hypoxic-ischemic encephalopathy: a randomized controlled trial. Arch Pediatr Adolesc Med 2011;165:692-700.

8 Jacobs SE, Berg M, Hunt R, Tarnow-Mordi WO, Inder TE, Davis PG: Cooling for newborns with hypoxic ischaemic encephalopathy. Cochrane Database Syst Rev 2013;1: CD003311.

9 Gunn AJ, Gluckman PD, Gunn TR: Selective head cooling in newborn infants after perinatal asphyxia: a safety study. Pediatrics 1998; 102:885-892.

10 Eicher DJ, Wagner CL, Katikaneni LP, Hulsey TC, Bass WT, Kaufman DA, Horgan MJ, Languani S, Bhatia JJ, Givelichian LM, Sankaran $\mathrm{K}$, Yager JY: Moderate hypothermia in neonatal encephalopathy: safety outcomes. Pediatr Neurol 2005;32:18-24.

11 Simbruner G, Mittal RA, Rohlmann F, Muche R; neo.nEURO.network Trial Participants: Systemic hypothermia after neonatal encephalopathy: outcomes of neo.nEURO.network RCT. Pediatrics 2010;126:e771-778.

12 Zhou WH, Cheng GQ, Shao XM, Liu XZ, Shan RB, Zhuang DY, Zhou CL, Du LZ, Cao Y, Yang Q, Wang LS; China Study Group: Selective head cooling with mild systemic hypothermia after neonatal hypoxic-ischemic encephalopathy: a multicenter randomized controlled trial in China. J Pediatr 2010;157: 367-372, 372.e361-e363.
13 Zhu C, Kang W, Xu F, Cheng X, Zhang Z, Jia L, Ji L, Guo X, Xiong H, Simbruner G, Blomgren K, Wang X: Erythropoietin improved neurologic outcomes in newborns with hypoxic-ischemic encephalopathy. Pediatrics 2009;124:e218-e226.

14 Elmahdy H, El-Mashad AR, El-Bahrawy H, El-Gohary T, El-Barbary A, Aly H: Human recombinant erythropoietin in asphyxia neonatorum: pilot trial. Pediatrics 2010;125:e1135e1142.

$15 \mathrm{Wu}$ YW, Bauer LA, Ballard RA, Ferriero DM, Glidden DV, Mayock DE, Chang T, Durand DJ, Song D, Bonifacio SL, Gonzalez FF, Glass HC, Juul SE: Erythropoietin for neuroprotection in neonatal encephalopathy: safety and pharmacokinetics. Pediatrics 2012;130:683691.

16 Wu YW, Mathur AM, Chang T, McKinstry RC, Mulkey SB, Mayock DE, Van Meurs KP, Rogers EE, Gonzalez FF, Comstock BA, Juul SE, Msall ME, Bonifacio SL, Glass HC, Massaro AN, Dong L, Tan KW, Heagerty PJ, Ballard RA: High-dose erythropoietin and hypothermia for hypoxic-ischemic encephalopathy: a phase II trial. Pediatrics 2016;137.

17 Juul SE, Aylward E, Richards T, McPherson RJ, Kuratani J, Burbacher TM: Prenatal cord clamping in newborn Macaca nemestrina: a model of perinatal asphyxia. Dev Neurosci 2007;29:311-320

18 Traudt CM, McPherson RJ, Bauer LA, Richards TL, Burbacher TM, McAdams RM, Juul SE: Concurrent erythropoietin and hypothermia treatment improve outcomes in a term nonhuman primate model of perinatal asphyxia. Dev Neurosci 2013;35:491-503.

19 Kattwinkel J (ed): NRP Neonatal Resuscitation Textbook, ed 6. Elk Grove Village/Dallas, American Heart Association/American Academy of Pediatrics, 2011.

20 Jacobson Misbe EN, Richards TL, McPherson RJ, Burbacher TM, Juul SE: Perinatal asphyxia in a nonhuman primate model. Dev Neurosci 2011;33:210-221.

21 Burbacher TM, Grant KS: Methods for studying nonhuman primates in neurobehavioral toxicology and teratology. Neurotoxicol Teratol 2000;22:475-486.

22 Smith SM, Jenkinson M, Johansen-Berg $H$, Rueckert D, Nichols TE, Mackay CE, Watkins KE, Ciccarelli O, Cader MZ, Matthews PM, Behrens TEJ: Tract-based spatial statistics: voxelwise analysis of multi-subject diffusion data. Neuroimage 2006;31:1487-1505.

23 Smith SM, Jenkinson M, Woolrich MW, Beckmann CF, Behrens TEJ, Johansen-Berg H, Bannister PR, De Luca M, Drobnjak I, Flitney DE, Niazy RK, Saunders J, Vickers J, Zhang Y, De Stefano N, Brady JM, Matthews PM: Advances in functional and structural MR image analysis and implementation as FSL. Neuroimage 2004;23(suppl 1):S208S219.
24 Smith SM: Fast robust automated brain extraction. Hum Brain Mapp 2002;17:143-155.

25 Andersson JLR, Jenkinson M, Smith S: Nonlinear optimisation. FMRIB technical report, TR07JA1, 2007. www.fmriboxacuk/analysis/ techrep.

26 Andersson JLR, Jenkinson M, Smith S: Nonlinear registration, aka Spatial normalisation. FMRIB technical report, TR07JA2, 2007. www.fmriboxacuk/analysis/techrep.

27 Rueckert D, Sonoda LI, Hayes C, Hill DL, Leach MO, Hawkes DJ: Nonrigid registration using free-form deformations: application to breast MR images. IEEE Trans Med Imaging 1999;18:712-721.

28 Smith SM, Jenkinson M, Johansen-Berg H, Rueckert D, Nichols TE, Mackay CE, Watkins KE, Ciccarelli O, Cader MZ, Matthews PM, Behrens TE: Tract-based spatial statistics: voxelwise analysis of multi-subject diffusion data. Neuroimage 2006;31:1487-1505.

29 Favrais G, van de Looij Y, Fleiss B, Ramanantsoa N, Bonnin P, Stoltenburg-Didinger G, Lacaud A, Saliba E, Dammann O, Gallego J, Sizonenko S, Hagberg H, Lelievre V, Gressens P: Systemic inflammation disrupts the developmental program of white matter. Ann Neurol 2011;70:550-565.

30 Ezzati M, Bainbridge A, Broad KD, Kawano G, Oliver-Taylor A, Rocha-Ferreira E, Alonso-Alconada D, Fierens I, Rostami J, Hassell KJ, Tachtsidis I, Gressens P, Hristova M, Bennett K, Lebon S, Fleiss B, Yellon D, Hausenloy DJ, Golay X, Robertson NJ: Immediate remote ischemic postconditioning after hypoxia ischemia in piglets protects cerebral white matter but not grey matter. J Cereb Blood Flow Metab 2016;36:1396-1411.

31 Fleiss B, Coleman HA, Castillo-Melendez M, Ireland Z, Walker DW, Parkington HC: Effects of birth asphyxia on neonatal hippocampal structure and function in the spiny mouse. Int J Dev Neurosci 2011;29:757-766.

32 Gressens P, Dingley J, Plaisant F, Porter H, Schwendimann L, Verney C, Tooley J, Thoresen M: Analysis of neuronal, glial, endothelial, axonal and apoptotic markers following moderate therapeutic hypothermia and anesthesia in the developing piglet brain. Brain Pathol 2008; 18:10-20.

33 Douaud G, Jbabdi S, Behrens TEJ, Menke RA, Gass A, Monsch AU, Rao A, Whitcher B, Kindlmann G, Matthews PM, Smith S: DTI measures in crossing-fibre areas: increased diffusion anisotropy reveals early white matter alteration in MCI and mild Alzheimer's disease. Neuroimage 2011;55:880-890.

34 Tsao H, Pannek K, Fiori S, Boyd RN, Rose S: Reduced integrity of sensorimotor projections traversing the posterior limb of the internal capsule in children with congenital hemiparesis. Res Dev Disabil 2014;35:250 260.
CP-Associated Neuropathological Changes in a Primate Model of HIE
Dev Neurosci 2017;39:124-140

DOI: $10.1159 / 000470903$ 
35 Rosenberger G, Nestor PG, Oh JS, Levitt JJ, Kindleman G, Bouix S, Fitzsimmons J, Niznikiewicz M, Westin CF, Kikinis R, McCarley RW, Shenton ME, Kubicki M: Anterior limb of the internal capsule in schizophrenia: a diffusion tensor tractography study. Brain Imaging Behav 2012;6:417-425.

36 Tusor N, Wusthoff C, Smee N, Merchant N, Arichi T, Allsop JM, Cowan FM, Azzopardi D, Edwards AD, Counsell SJ: Prediction of neurodevelopmental outcome after hypoxicischemic encephalopathy treated with hypothermia by diffusion tensor imaging analyzed using tract-based spatial statistics. Pediatr Res 2012;72:63-69.

37 Billiards SS, Haynes RL, Folkerth RD, Borenstein NS, Trachtenberg FL, Rowitch DH, Ligon KL, Volpe JJ, Kinney HC: Myelin abnormalities without oligodendrocyte loss in periventricular leukomalacia. Brain Pathol 2008; 18:153-163.

38 Delcour M, Olivier P, Chambon C, Pansiot J, Russier M, Liberge M, Xin D, Gestreau C, Alescio-Lautier B, Gressens P, Verney C, Barbe MF, Baud O, Coq JO: Neuroanatomical, sensorimotor and cognitive deficits in adult rats with white matter injury following prenatal ischemia. Brain Pathol 2012;22:116.

39 Shrivastava K, Chertoff M, Llovera G, Recasens M, Acarin L: Short and long-term analysis and comparison of neurodegeneration and inflammatory cell response in the ipsilateral and contralateral hemisphere of the neonatal mouse brain after hypoxia/ischemia. Neurol Res Int 2012;2012:781512.

40 Fleiss B, Gressens P: Tertiary mechanisms of brain damage: a new hope for treatment of cerebral palsy? Lancet Neurol 2012;11:556-566.

41 Bilbo SD, Barrientos RM, Eads AS, Northcutt A, Watkins LR, Rudy JW, Maier SF: Early-life infection leads to altered BDNF and IL- $1 \beta$ mRNA expression in rat hippocampus following learning in adulthood. Brain Behav Immun 2008;22:451-455.

42 Reinebrant HE, Wixey JA, Buller KM: Disruption of raphe serotonergic neural projections to the cortex: a potential pathway contributing to remote loss of brainstem neurons following neonatal hypoxic-ischemic brain injury. Eur J Neurosci 2012;36:3483-3491.

43 Bellot B, Peyronnet-Roux J, Gire C, Simeoni U, Vinay L, Viemari JC: Deficits of brainstem and spinal cord functions after neonatal hypoxia-ischemia in mice. Pediatr Res 2014;75: 723-730.

44 Surveillance of cerebral palsy in Europe: a collaboration of cerebral palsy surveys and registers. Dev Med Child Neurol 2000;42:816-824.

45 Johnston MV, Hagberg H: Sex and the pathogenesis of cerebral palsy. Dev Med Child Neurol 2007;49:74-78.

46 Du L, Bayir H, Lai Y, Zhang X, Kochanek PM, Watkins SC, Graham SH, Clark RS: Innate gender-based proclivity in response to cytotoxicity and programmed cell death pathway. J Biol Chem 2004;279:38563-38570.

47 Cerghet M, Skoff RP, Swamydas M, Bessert D: Sexual dimorphism in the white matter of rodents. J Neurol Sci 2009;286:76-80.

48 Pappas A, Korzeniewski SJ: Long-term cognitive outcomes of birth asphyxia and the contribution of identified perinatal asphyxia to cerebral palsy. Clin Perinatol 2016;43:559-572.

49 Roth GS, Mattison JA, Ottinger MA, Chachich ME, Lane MA, Ingram DK: Aging in rhesus monkeys: relevance to human health interventions. Science 2004;305:1423-1426.

50 Shankaran S, Laptook AR, Pappas A, McDonald SA, Das A, et al; Eunice Kennedy Shriver National Institute of Child Health; Human Development Neonatal Research
Network: Effect of depth and duration of cooling on deaths in the NICU among neonates with hypoxic ischemic encephalopathy: a randomized clinical trial. JAMA 2014;312: 2629-2639.

51 Miller SP, Ramaswamy V, Michelson D, Barkovich AJ, Holshouser B, Wycliffe N, Glidden DV, Deming D, Partridge JC, Wu YW, Ashwal S, Ferriero DM: Patterns of brain injury in term neonatal encephalopathy. J Pediatr 2005;146:453-460.

52 Bax M, Tydeman C, Flodmark O: Clinical and MRI correlates of cerebral palsy: the European Cerebral Palsy Study. JAMA 2006;296: 1602-1608.

53 Numata Y, Onuma A, Kobayashi Y, Sato-Shirai I, Tanaka S, Kobayashi S, Wakusawa K, Inui $\mathrm{T}$, Kure $\mathrm{S}$, Haginoya $\mathrm{K}$ : Brain magnetic resonance imaging and motor and intellectual functioning in 86 patients born at term with spastic diplegia. Dev Med Child Neurol 2013;55:167-172.

54 Benini R, Dagenais L, Shevell MI; Registre de la Paralysie Cérébrale au Québec Consortium: Normal imaging in patients with cerebral palsy: what does it tell us? J Pediatr 2013; 162:369-374 e361.

55 Rose S, Guzzetta A, Pannek K, Boyd R: MRI structural connectivity, disruption of primary sensorimotor pathways, and hand function in cerebral palsy. Brain Connect 2011;1:309316.

56 Johansson BB: Functional outcome in rats transferred to an enriched environment 15 days after focal brain ischemia. Stroke 1996; $27: 324-326$

57 Will B, Galani R, Kelche C, Rosenzweig MR: Recovery from brain injury in animals: relative efficacy of environmental enrichment, physical exercise or formal training (19902002). Prog Neurobiol 2004;72:167-182. 